\title{
Ion Implantation and Transient Melting: A New Approach to Formation of Superconducting $\mathrm{MgB}_{2}$ Phases
}

\author{
J. Piekoszewski ${ }^{a, d}$, W. Kempinśíl ${ }^{b}$, J. Stankowski $^{b}$, \\ F. Prokert ${ }^{c}$, E. Richter ${ }^{c}$, J. StaniseaWski ${ }^{a}$, Z. Werner ${ }^{a, e}$ \\ AND W. SZYMCZYK ${ }^{a}$ \\ ${ }^{a}$ The Andrzej Sołtan Institute for Nuclear Studies \\ 05-400 Otwock/Świerk, Poland \\ ${ }^{b}$ Institute of Molecular Physics, Polish Academy of Sciences \\ M. Smoluchowskiego 17, 60-179 Poznań, Poland \\ ${ }^{c}$ Forschungszentrum Rossendorf e.V. Institut für Ionenstrahlphysik \\ und Materialforschung, Postfach 510119, 01314 Dresden, Germany \\ ${ }^{d}$ Institute of Nuclear Chemistry and Technology \\ Dorodna 16, 03-145 Warszawa, Poland \\ ${ }^{e}$ Institute of Physical Chemistry, Polish Academy of Sciences \\ M. Kasprzaka 44/52, 01-224 Warszawa, Poland
}

(Received April 5, 2004; revised version August 9, 2004)

An attempt to synthesize superconducting $\mathrm{MgB}_{2}$ inter-metallic compound from the liquid state is presented. The process consists of two steps. In the first one, boron ions are implanted into a magnesium substrate. In the second one, the near-surface region of such system is melted by short, intense hydrogen plasma pulses without necessity of annealing in $\mathrm{Mg}$ vapor. A magnetically modulated microwave absorption method was used to detect superconducting regions in the obtained $\mathrm{MgB}_{2}$ layer. Percolation between nano-regions (islands) of $\mathrm{MgB}_{2}$ has not been observed. However, a superconducting state of the insulated islands has been experimentally proven with transition temperatures $T_{\mathrm{C}}$ as high as $31 \mathrm{~K}$.

PACS numbers: 74.70.-b, 74.78.--w 


\section{Introduction}

Superconductivity in $\mathrm{MgB}_{2}$ inter-metallic compound at a temperature as high as $T_{\mathrm{C}}=39 \mathrm{~K}$ was discovered by Nagamatsu et al. [1] in 2001. This discovery stirred the research community and attracted a great deal of interest. Exploration of the new material started in two directions: (i) development of new methods of fabricating and improving the quality of bulk $\mathrm{MgB}_{2}$ and (ii) the same as above, in the area of thin $\mathrm{MgB}_{2}$ films formed on various substrates. The present study belongs to the latter direction. Encouraging results obtained in this field were widely reported in the literature, e.g. [2,3]. A common feature of the approaches presented so far is that superconducting $\mathrm{MgB}_{2}$ films are formed in the solid phase in the $\mathrm{Mg}-\mathrm{B}$ system by suitable thermal treatment procedures. In the present work we attempted to synthesize superconducting $\mathrm{MgB}_{2}$ phase from the liquid state using a transient melting process (TMP) without necessity of annealing in $\mathrm{Mg}$ vapors. A magnetically modulated microwave absorption method was used to detect local superconducting regions in $\mathrm{MgB}_{2}$ thin films.

\section{Key concept}

Our idea is to verify two new approaches to formation of thin (nano-scale) superconducting $\mathrm{MgB}_{2}$ layers. They are:

(i) High-dose boron ion implantation into magnesium substrate followed by transient melting the near-surface layer with high intensity plasma pulses. In principle, the TMP can be accomplished also by other means, including pulsed lasers, electron/ion beams, flash lamp annealing (FLA) processes, etc.

(ii) High-dose magnesium implantation into a boron film grown on an appropriate substrate (preferably silicon) followed by the TMP as in (i).

In the present work we focused our attention on the former approach with the use of pulsed plasma beams.

In order to get insight into heat evolution within the substrate subjected to pulse plasma treatment we calculated the course of temperature vs. time and melt depth vs. time in pure $\mathrm{Mg}$ irradiated with plasma pulses of a few $\mathrm{J} / \mathrm{cm}^{2}$ energy density. The simulations were performed using the ETLIT computer code described in Ref. [4]. A sample result is shown in Fig. 1. For $2 \mathrm{~J} / \mathrm{cm}^{2}$ the molten phase lasts about $1.5 \mu \mathrm{s}$ and its front reaches the depth of about $2.2 \mu \mathrm{m}$. The cooling rate after solidification of the near-surface region is of the order of $10^{7} \mathrm{~K} / \mathrm{s}$. These results can only be treated as a rough estimation since the calculations have been performed for pure $\mathrm{Mg}$, whereas the actual samples contain also a strongly modified (boron implanted) material. 


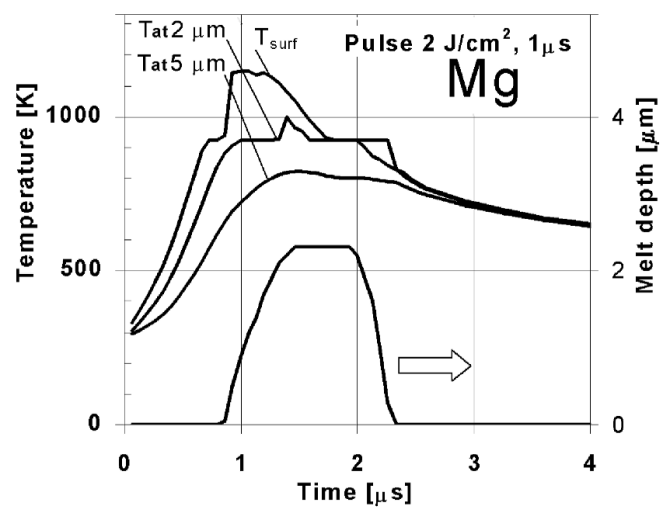

Fig. 1. Calculated time dependence of the temperature at different depths and the position of the melt-front in pure $\mathrm{Mg}$ after irradiation with a plasma pulse.

\section{Experimental}

\subsection{Sample preparation}

The samples were cut out from a commercial magnesium ingot of $99.8 \mathrm{wt} \%$ purity in the form of highly polished discs of $3.8 \mathrm{~mm}$ and $20 \mathrm{~mm}$ diameter. They were implanted with $100 \mathrm{keV}$ boron ions to the dose of $5 \times 10^{18} \mathrm{~B} / \mathrm{cm}^{2}$ using a Danfysik Model 1009-200 implanter. During implantation the samples were kept at a temperature of $200-250^{\circ} \mathrm{C}$. A subsequent step of the adopted procedure was the transient melting process using high intensity pulsed plasma beams (HIPPBs). The plasma pulses were generated in a rod plasma injector (IBIS) type of accelerator described elsewhere [5]. Briefly, the plasma pulses are generated as a result of a low-pressure, high-current discharge between two concentric sets of electrodes. High voltage pulses ignite the discharge producing the plasma pulses delayed for a time $\tau_{\mathrm{D}}$ with respect to the moment of injection of the working gas into the inter-electrode space. A power level in the range of several $\mathrm{MW} / \mathrm{cm}^{2}$ and pulse duration in the $\mu$ s scale are sufficient to raise the temperature of the near-surface layer of most solids up to their melting point (or above). When this region is molten, a rapid inward diffusion of the pulse-delivered and/or pre-deposited atoms into the liquid can occur, leading to the formation of new phases, alloys or compounds. If $\tau_{\mathrm{D}}$ is set long enough to allow the injected gas to expand over the whole interelectrode space, the plasma pulse contains almost exclusively the elements of the working gas. The conditions of the HIPPBs treatment in the present experiments were as follows:

- plasma pulse energy densities: between $2 \mathrm{~J} / \mathrm{cm}^{2}$ and $6 \mathrm{~J} / \mathrm{cm}^{2}$

- working gas: hydrogen

- number of pulses: from 1 to 4

- pulse duration: about $1 \mu \mathrm{s}$. 


\subsection{X-ray diffraction}

The X-ray diffraction patterns were obtained in the grazing incidence technique using a standard instrument (D5000, Bruker AXS) equipped with a thin film setup composed of a collimator and a secondary monochromator placed in front of the scintillation detector. At the used $\mathrm{Cu} K_{\alpha}$ radiation and the applied incidence angle of $1^{\circ}$, the $1 / e$ penetration depth of the X-rays amounts to approximately $300 \mathrm{~nm}$. Thus the recorded signals originate mainly in the pulse-modified thin surface layer, whereas the input from the magnesium substrate is diminished. The phase analysis was carried out using the PDF database implemented in the Bruker AXS evaluation code.

\subsection{Superconducting state detection - MMMA}

Microwave non-resonant dissipation observed around zero field ("EPR zero-field line") is considered as a convenient method in characterizing superconducting state. Since the discovery of high temperature superconductors (HTS), the magnetically modulated microwave absorption (MMMA) technique has been successfully established [6]. The MMMA signal in granular superconducting samples as well as in point superconductor-insulator-superconductor (SC-I-SC) weak contacts is the result of Josephson dissipation related to the fluctuation of Cooper pairs to electrons in the conducting band. A Josephson junctions system (JJS) can be considered as a separate phase embedded in the host surrounding. MMMA is a unique and a very sensitive contact-less method to determine $T_{\mathrm{C}}$ for small amounts of SC phases dispersed below the percolation threshold. MMMA has been applied already to study intercalated fullerene, perovskite-composite (superconductorinsulator), as well as $\mathrm{MgB}_{2}$.

Thin films of $\mathrm{MgB}_{2}$ superconductor sample were placed in a microwave resonator of the EPR spectrometer with modulation of the sweeping magnetic field. In this procedure, the output signal has a differential form of absorbed power $\mathrm{d} P / \mathrm{d} B[7]$. A weak Josephson contact $\mathrm{SC}-\mathrm{I}-\mathrm{SC}$ is a basic element that interacts magnetically with microwave field. The Josephson dissipation related to Cooper pairs breaking leads to voltage fluctuations like in the EPR spectroscopy. The Josephson hysteresis loop (JHL) is the confirmation that the zero-field MMMA line is related to the superconducting state. The JHL has an opposite circulation vs. magnetic field $B$ in comparison to a typical magnetic hysteresis loop.

\section{Results and discussion}

\subsection{X-ray diffraction}

Figure 2 presents grazing angle $\left(\omega=1^{\circ}\right)$ X-ray diffraction (XRD) patterns for an as-implanted sample and for samples treated after implantation with various number of pulses (2 and 4$)$ of different energy densities $\left(2.85 \mathrm{~J} / \mathrm{cm}, 5.2 \mathrm{~J} / \mathrm{cm}^{2}\right.$, and 
$5.65 \mathrm{~J} / \mathrm{cm}^{2}$ ). Two regularities are clearly seen. Firstly, regardless of the number of pulses, the $\mathrm{MgB}_{2}$ (101) peak has a higher intensity and is better developed for a lower pulse energy density, i.e. for $2.85 \mathrm{~J} / \mathrm{cm}^{2}$ (patterns $\mathrm{b}$ and e) than for $5.2 \mathrm{~J} / \mathrm{cm}^{2}$ and $5.65 \mathrm{~J} / \mathrm{cm}^{2}$ (patterns c and d). Secondly, the $\mathrm{MgB}_{2}$ (101) peak for the pulse-treated samples is shifted towards the greater values of $2 \theta$ with respect to the one for the as-implanted sample. In $\mathrm{MgB}_{2}$ films in which superconductivity was achieved by furnace annealing after ion implantation [8], the (101) peak was shifted towards greater $2 \theta$ like in our case.

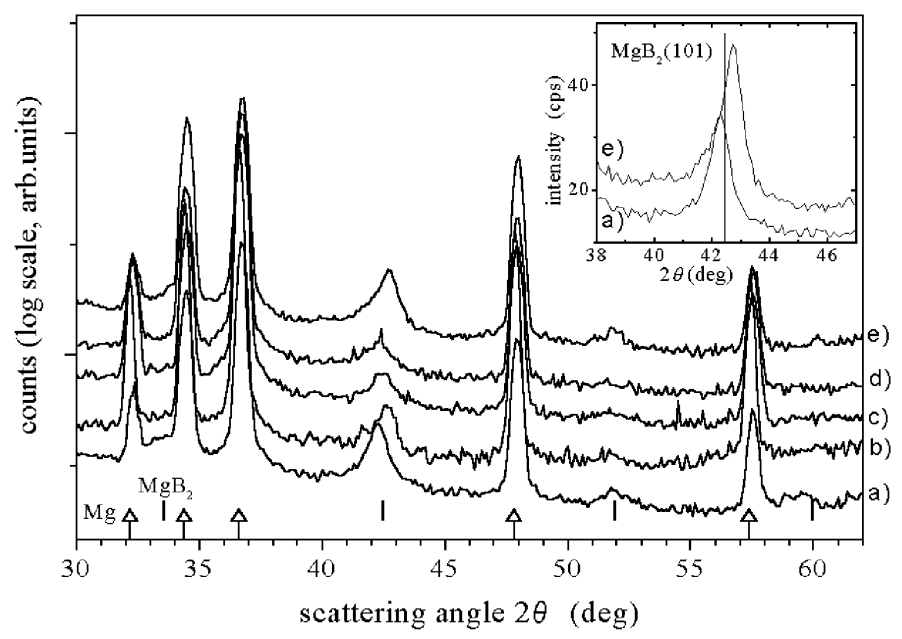

Fig. 2. XRD patterns for boron implanted and hydrogen pulse plasma treated $\mathrm{Mg}$ substrate: a) as-implanted, b) 4 pulses, $2.85 \mathrm{~J} / \mathrm{cm}^{2}$, c) 2 pulses, $5.65 \mathrm{~J} / \mathrm{cm}^{2}$, d) 4 pulses, $5.2 \mathrm{~J} / \mathrm{cm}^{2}$, e) 2 pulses, $2.85 \mathrm{~J} / \mathrm{cm}^{2}$.

A careful examination of our XRD data (average taken over 5 samples) indicates that the $a$ lattice constant decreased in the pulse-treated samples with respect to the as-implanted samples by $\Delta a / a=-0.71 \%$, whereas the $c$ lattice constant increased by $\Delta c / c=0.37 \%$. According to theoretical predictions given by Wan et al. [9] such changes should lead to an increased density of states near the Fermi level and therefore to an increased $T_{\mathrm{C}}$. On the other hand, in Ref. [10] the highest value of the transition temperature $T_{\mathrm{C}}$ was observed for the sample, in which the $c$ lattice constant (calculated from the $\mathrm{MgB}_{2}$ (002) peak position) had the smallest value with respect to the bulk data $(\Delta c / c=-0.2 \%)$. Besides, some authors claim that $T_{\mathrm{C}}$ rises with lattice expansion [11-13]. Therefore, we feel it would be premature for us to deduce the superconducting properties from our experimental XRD result.

The presence of $\mathrm{MgB}_{2}$ phase in the as-implanted samples suggests a priori that there are three possible ways the superconducting state might develop in the studied samples: (i) it appears as a result of boron implantation only and plasma 
irradiation does not improve it; (ii) it appears as a result of boron implantation but the irradiation by plasma pulses is necessary to enhance its properties; (iii) the boron-implanted sample is not superconducting down to the lowest temperature within our experimental range and pulse plasma irradiation is necessary to get the superconducting state. To check which of the above listed possibilities takes place, we have performed the MMMA measurements.

\section{2. $M M M A$}

Figure 3 shows how the samples were positioned in the region of the maximum magnetic $H_{1}^{\sim}$ microwave field. From this figure one can see that the MMMA signal in the studied thin layer region appears only when a large enough part of the $\mathrm{MgB}_{2}$ thin film absorbs the energy of the microwave field.

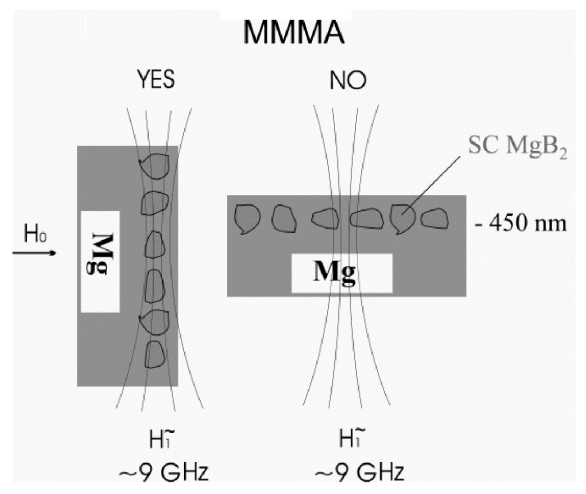

Fig. 3. Position of the thin layer of the sample in respect to magnetic component of the microwave field.

No trace of hysteresis loop was observed on as-implanted samples even at a temperature as low as $4.2 \mathrm{~K}$. Thus the possibility (i) and (ii) of the three above listed ones must be excluded.

Superconductivity has been confirmed for two samples of the $\mathrm{MgB}_{2}$ nano-regions. Below $20 \mathrm{~K}$ weak superconducting MMMA signals versus temperature (or versus the magnetic field) appear for sample labeled "44", treated with hydrogen plasma pulses of $1.9 \mathrm{~J} / \mathrm{cm}^{2}$ energy density. JHL hysteresis loop and MMMA signal vs. temperature are shown in Fig. $4 \mathrm{a}$ and $\mathrm{b}$, respectively. The $T_{\mathrm{C}}$ temperature is marked with an arrow in Fig. 4b. MMMA signals observed for sample "49" treated with melting pulses of $3.0 \mathrm{~J} / \mathrm{cm}^{2}$ energy density are shown in Fig. 5a and b. In this case $T_{\mathrm{C}}$ amounts to $31 \mathrm{~K}$.

MMMA signals observed on similar volumes of samples of a $\mathrm{MgB}_{2}$ powder produced by the standard powder/powder annealing method [1] are shown for comparison in Figs. 6 and 7. They are larger by three orders of magnitude than those shown in Figs. 4 and 5. 


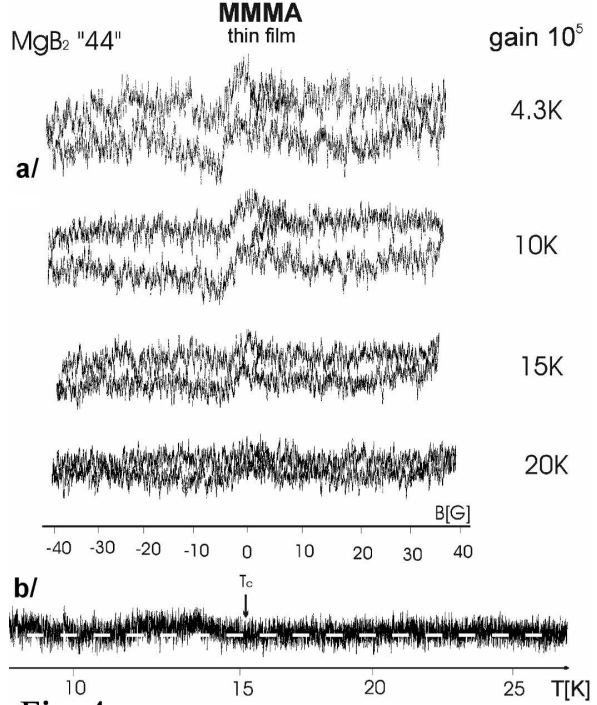

Fig. 4
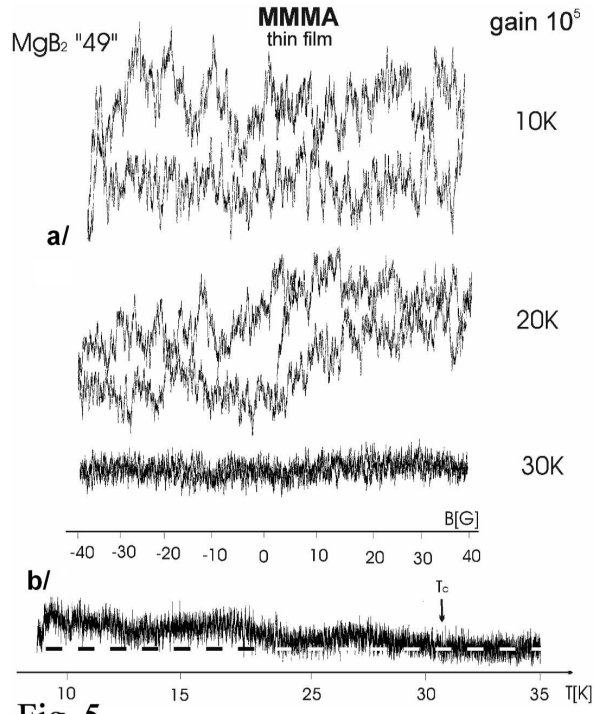

Fig. 5

Fig. 4. MMMA signal versus external magnetic field $B$ (a) and temperature (b) for the sample " 44 ".

Fig. 5. MMMA signal versus external magnetic field $B$ (a) and temperature (b) for the sample " 49 ".
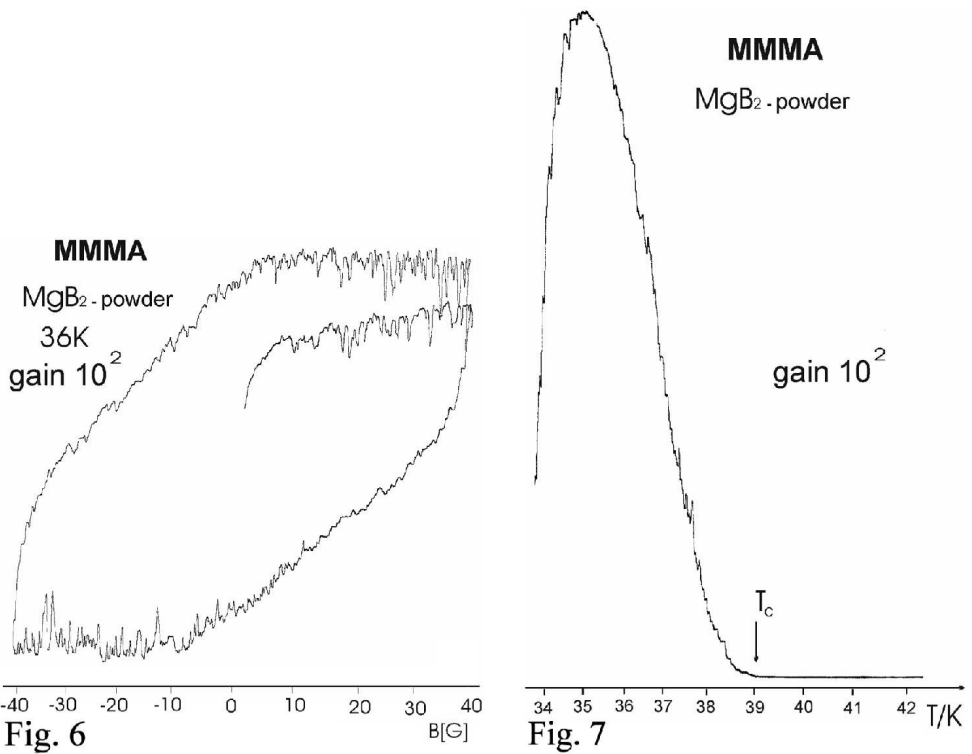

Fig. 6. MMMA signal versus external magnetic field $B$ for a powder sample.

Fig. 7. MMMA signal versus temperature for a powder sample. 
JHL loops shown in Figs. 4 and 5 confirm that quantum fluxes can penetrate the superconducting islands, which seem to be mostly separated regions with a low level of Josephson junctions between them. This is a reason why the MMMA signals versus temperature are very weak in comparison to the case of the powdered sample (Fig. 7).

\section{Conclusions}

First attempts to apply a new technology of producing superconducting $\mathrm{MgB}_{2}$ layers have yielded very promising results. Even if percolation between islands of the $\mathrm{MgB}_{2}$ nano-regions created within the $\mathrm{Mg}$ substrate has not been obtained, a superconducting state of the islands has been experimentally proven. Work on obtaining continuous $\mathrm{MgB}_{2}$ layers on the host $\mathrm{Mg}$ substrate is under way.

\section{References}

[1] J. Nagamatsu, N. Nakagawa, T. Muranaka, Y. Zenitani, J. Akimitsu, Nature 410, 63 (2001).

[2] G. Bumel, D.J. Kang, H.N. Lee, S.H. Moon, B. Oh, Appl. Phys Lett. 79, 3464 (2001).

[3] S. Schiestel, C.A. Carosella, J.S. Horowitz, M. Osofsky, C. Kendziora, S.B. Qadri, D.L. Knies, Surf. Coat. Technol. 158, 203 (2002).

[4] W. Szyszko, D.Sc. Thesis, UMCS Lublin University, Lublin 1998.

[5] J. Piekoszewski, J. Langner, Nucl. Instrum. Methods B 53, 148 (1991).

[6] R. Durny, J. Hautala, S. Ducharme, B. Lee, O.G. Symko, P.C. Taylor, D.J. Zheng, J.A. Xu Phys. Rev. B 36, 2361 (1987); J. Stankowski, P.K. Kahol, N.S. Dalal, J.S. Moodera, ibid. p. 7126; K.W. Blazey, K.A. Mueller, J.G. Bednorz, W. Berlinger, G. Amoretti, E. Buluggiu, A. Vera, F.C. Matacotta, ibid. p. 7241.

[7] J. Stankowski. B. Czyżak, J. Martinek, B. Andrzejewski, in: Recent Developments in HTSC, Proc. 1st Polish-US Conf. Wroctaw, Duszniki Zdrój (Poland) September 1995, Eds. J. Klamut, B.W. Veal, B.M. Dąbrowski, P.W. Klamut, M. Kazimierski, Springer, New York 1996, p. 167.

[8] H.Y. Zhai, H.M. Christen, C.W. White, J.D. Budai, D.H. Lowndes, Appl. Phys. Lett. 80, 4786 (2002).

[9] X. Wan, J. Dong, H. Weng, D.Y. Xing, cond-mat/0104216v3 (2001).

[10] H. Yamazaki, Y. Hikita, H. Hori, H. Takagi, Appl. Phys Lett. 83, 3740 (2003).

[11] N. Hur, P.A. Scharma, S. Guha, M.Z. Cieplak, D.J. Werder, Y. Horibe, C.H. Chen, S.W. Choeng, Appl. Phys. Lett. 79, 4180 (2001).

[12] J. Tang, L.-Ch. Qin, A. Matsushita, Y. Takano, K. Togano, H. Kito, H. Igara, Phys. Rev. 64, 132509-1 (2001).

[13] J.B. Neaton, A. Perali, cond-mat/0104098v1 (2001). 